Itinéraires Itinéraires

Littérature, textes, cultures

2015-3 | 2016

Ethos numériques

\title{
L'ethos discursif et le défi du Web
}

Discursive Ethos and the Challenge of the Web

Dominique Maingueneau

\section{OpenEdition}

Journals

Édition électronique

URL : http://journals.openedition.org/itineraires/3000

DOI : $10.4000 /$ itineraires.3000

ISSN : 2427-920X

Éditeur

Pléiade

\section{Référence électronique}

Dominique Maingueneau, «L'ethos discursif et le défı du Web », Itinéraires [En ligne], 2015-3 | 2016, mis en ligne le 01 juillet 2016, consulté le 19 avril 2019. URL : http://journals.openedition.org/ itineraires/3000 ; DOI : 10.4000/itineraires.3000

Ce document a été généré automatiquement le 19 avril 2019

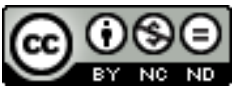

Itinéraires est mis à disposition selon les termes de la licence Creative Commons Attribution - Pas d'Utilisation Commerciale - Pas de Modification 4.0 International. 


\title{
L'ethos discursif et le défi du Web
}

\author{
Discursive Ethos and the Challenge of the Web
}

\section{Dominique Maingueneau}

1 L'un des problèmes que pose l'ethos considéré comme outil d'analyse est que la conception que l'on s'en fait dépend pour une bonne part des types de discours auxquels on l'applique de manière privilégiée. C'est ce que j'ai essayé de montrer récemment (Maingueneau 2014) en prenant pour données des productions de locuteurs non experts, données qui tranchent sur les corpus habituels des analyses menées en termes d'ethos. Cette difficulté est encore plus évidente quand on a affaire au Web, dont le fonctionnement ne ressortit pas au régime de discours dans et pour lequel la notion d'ethos a été élaborée, en rhétorique comme en analyse du discours. Ce nouveau type de matériau, qui pose la question essentielle de l'«identité numérique » (Perea 2010), ne peut qu'avoir une incidence sur la notion même d'ethos.

\section{L'ethos discursif}

2 La notion même d'ethos est loin d'être évidente. Je signalerai deux difficultés. La première est que ce n'est pas « un concept théorique clair » (Auchlin 2001: 93) mais une notion qui s'appuie sur le sens commun: en énonçant, tout locuteur active nécessairement chez l'interprète la construction d'une certaine représentation de luimême, qu'il doit s'efforcer de contrôler. Pour en faire un concept opératoire, il faut l'inscrire dans une problématique qui n'en exploite que certaines facettes, en fonction de la discipline et des présupposés du chercheur. La seconde difficulté tient au glissement constant de l'ethos discursif à l'ethos non discursif. La notion d'ethos est foncièrement hybride, comme d'autres en analyse du discours - à commencer par celles de « discours » ou de « genre de discours »- et cela lui confère une instabilité essentielle. Ce glissement est déjà visible dans l'œuvre d'Aristote. On cite souvent le passage de la Rhétorique où Aristote définit l'ethos proprement discursif : « on persuade par le caractère quand le discours est de nature à rendre l'orateur digne de foi [...] Mais il faut que cette confiance soit l'effet du discours, non d'une prévention sur le caractère de l'orateur ${ }^{1}{ }^{\prime}$ (1356 a). Pourtant, 
comme l'a bien montré F. Woerther (2007), dans la même Rhétorique et dans d'autres ouvrages d'Aristote (sur la musique, la politique, la zoologie, etc.), la notion d'ethos est utilisée pour décrire des phénomènes qui ne sont pas du ressort des sciences du langage. Beaucoup plus près de nous, il existe en sociologie une réflexion sur l'ethos illustrée par des noms tels que Max Weber, Norbert Elias ou Pierre Bourdieu; la microsociologie de Goffman et sa "présentation de soi " constitue une sorte de pont entre les deux traditions, rhétorique et sociologique. C'est particulièrement évident dans le livre de R. Amossy (2011), au titre révélateur : La Présentation de soi. Ethos et identité verbale.

3 Pour ma part, c'est en analyste du discours que j'aborde l'ethos, par conséquent en termes d'ethos discursif. Celui-ci implique à la fois l'ethos dit (ce que le locuteur dit sur lui-même, par exemple qu'il est un homme simple), et l'ethos montré (ce que montre sa manière d'énoncer). Cette distinction est exprimée chez Ducrot (1984: 201) à travers l'opposition entre locuteur- $L$ (le locuteur en tant qu'il est en train d'énoncer) et locuteur- $\lambda$ (le locuteur en tant qu'être du monde, hors de l'énonciation). L'ethos discursif se montre dans l'acte d'énonciation, il ne se dit pas dans l'énoncé; il est perçu, mais il ne fait pas l'objet du discours. Alors que l'ethos discursif est une dimension constitutive de toute énonciation, l'ethos dit, lui, n'est pas obligatoire. Ducrot n'évoque que l'ethos dit non discursif, celui qui concerne la personnalité du locuteur ; mais il existe aussi un ethos dit verbal, c'est-à-dire qui porte sur les propriétés de l'énonciation elle-même ( «je ne sais pas m'exprimer en public », « je n'aime pas les longs discours », etc.).

4 Les analystes du discours distinguent en outre «ethos discursif » et "ethos préalable » (ou « prédiscursif »), car bien souvent les destinataires disposent d'une représentation du locuteur antérieure à sa prise de parole, surtout s'il s'agit de personnes qui occupent la scène médiatique. Pour le Web les choses sont beaucoup plus compliquées, étant donné que bien souvent les intervenants sont invisibles (ainsi les concepteurs d'un site), anonymes ou pseudonymes.

5 Mais ce que recouvre l'ethos discursif est loin d'être clair. Quand on parcourt les travaux qui en font usage, on constate que son contenu est instable. Il est par exemple question d'ethos "de paysan", "de femme ", "d'expert», etc., ou encore d'ethos "calme», " communiste ", « rural », " prophétique », etc. Pour introduire un minimum d'ordre, j'ai proposé (Maingueneau 2014) de spécifier davantage en distinguant dans l'ethos trois dimensions (catégorielle, expérientielle et idéologique) qui sont plus ou moins saillantes selon les textes considérés.

1. La dimension «catégorielle » recouvre des rôles discursifs ou des statuts extradiscursifs. Les rôles discursifs sont ceux qui sont liés à l'activité de parole: animateur, conteur, prédicateur, etc. Les statuts extradiscursifs peuvent être de natures très variées : père de famille, fonctionnaire, médecin, villageois, Américain, célibataire, etc.

2. La dimension «expérientielle» recouvre les caractérisations socio-psychologiques stéréotypiques : bon sens et lenteur du campagnard, dynamisme du jeune cadre, etc.

3. Une dimension «idéologique » renvoie à des positionnements dans un champ : féministe, de gauche, conservateur ou anticlérical, dans le champ politique, romantique, surréaliste ou naturaliste, dans le champ littéraire, etc.

6 Ces trois dimensions interagissent fortement. Par exemple, le paysan (catégoriel) a des affinités stéréotypiques avec le bon sens (expérientiel) et le conservatisme (idéologique), l'expert avec le calme et la neutralité, etc. 
$7 \quad$ A priori, la liste des prédicats qu'on peut prendre en compte pour caractériser un ethos est ouverte. Mais la plupart du temps, en fonction du genre et du type de discours concerné, l'analyste filtre de manière drastique les éléments qu'il juge pertinents. S'il aborde un genre politique électoral, il va plutôt privilégier les prédicats qui ressortissent au positionnement idéologique (de droite, pro-européen, anarchiste, etc.) et certains prédicats psychologiques (compétence, autorité, honnêteté, courage, etc.). C'est ainsi que P. Charaudeau (2005) a essayé de dégager les catégories d'ethos qui seraient spécifiques des acteurs politiques. Il les regroupe autour de deux axes: «crédibilité» et «identification ». Le premier, par exemple, recouvre des prédicats expérientiels tels que " sérieux », " vertueux », « compétent », etc.

Dans mes propres travaux j'ai mis l'accent sur l'ethos expérientiel, dans la mesure où j'ai élaboré ma conception de l'ethos en étudiant des corpus religieux (Maingueneau 1984), puis littéraires (1993) et publicitaires (1998), où la relation entre la parole et un imaginaire du corps est forte. Dans ce modèle, le destinataire construit la figure d'un garant doué de propriétés physiques (corporalité) et psychologiques (caractère) en s'appuyant sur un ensemble diffus de représentations sociales stéréotypiques évaluées positivement ou négativement, que l'énonciation contribue à conforter ou à transformer. Le pouvoir de persuasion d'un discours tient ainsi pour une bonne part au fait qu'il amène le destinataire à s'identifier au mouvement d'un corps, fût-il très schématique, investi de valeurs historiquement spécifiées. Les «idées » suscitent l'adhésion du lecteur parce que la manière de dire implique une manière d'être. Cette conception de l'ethos est mise en évidence à travers le concept d' "incorporation ", qui joue sur trois registres :

- l'énonciation confère une « corporalité » au garant, elle lui donne corps ;

- le destinataire incorpore, assimile à travers l'énonciation un ensemble de schèmes qui correspondent à une manière spécifique de se rapporter au monde ;

- ces deux premières incorporations permettent la constitution d'un corps, de la communauté imaginaire de ceux qui adhèrent au même discours.

9 L'incorporation du destinataire implique un «monde éthique » (Maingueneau 2000) dont participe le garant. Ce «monde éthique» subsume un certain nombre de situations stéréotypiques associées à des comportements verbaux et non verbaux (le monde éthique du cadre dynamique, des snobs, des stars de cinéma, etc.).

Quelle que soit la conception que l'on se fait de l'ethos, on ne peut l'autonomiser. Il n'est qu'une dimension de la scène d'énonciation. Si l'on admet notre tripartition en scène englobante / scène générique / scénographie (Maingueneau 1998), l'ethos opère sur ces trois niveaux, qui interagissent. Par exemple, dans un entretien télévisé entre un journaliste et un homme ou une femme politique, la scène englobante (en l'occurrence le discours politique) va appeler de la part de l'interviewé des catégories d'ethos du type de celles décrites par Charaudeau. Au niveau de l'entretien télévisé aussi, de la scène générique, interviennent des contraintes spécifiques: être clair, courtois, etc. À cela s'ajoutent les contraintes liées au positionnement idéologique (un homme ou une femme d'un parti de gauche doit montrer qu'il est un homme ou une femme de gauche), qui doit être compatible avec la scénographie choisie dans cet entretien: relation intimiste, monologue oratoire, etc. 


\section{Ce que modifie Internet}

11 Le développement d'Internet a mis en cause la conception du genre de discours qui sert de base implicite à la plupart des travaux sur l'ethos (Maingueneau 2013). Cette conception classique du genre est structurée par la hiérarchie scène englobante > scène générique > scénographie, où la scène générique joue le rôle de pivot. Dans cette configuration, l'hypergenre ${ }^{2}$ est périphérique. Sur Internet la situation est bien différente. Dans la mesure où on a affaire à des sites web partageant un ensemble de contraintes d'ordre technique, il se produit une homogénéisation des productions. Dès lors, ce sont la scénographie et l'hypergenre qui passent au premier plan. Les sites se laissent en effet diviser en grandes catégories telles que "sites marchands ", «blogs", " sites d'information », etc., qui n'ont pas les caractéristiques de genres de discours, mais plutôt d'hypergenres, à l'intérieur desquels s'élaborent des scénographies très variées. Le principal problème de ceux qui élaborent et gèrent les sites est de définir des choix en matière de mise en scène de l'énonciation, en mobilisant à la fois des ressources verbales et multimodales (image fixe, mouvante, son) et des opérations hypertextuelles.

12 En fait, la notion même de scénographie doit être complexifiée pour rendre raison des spécificités du Web, où la scénographie est double : verbale et numérique. La scénographie "verbale » est celle qu'impliquent les énoncés qui apparaissent sur l'écran; quant à la scénographie « numérique », elle comporte deux dimensions :

- iconotextuelle (le site contient des images, et il se présente lui-même comme un ensemble d'images bornées par l'écran);

- réticulaire : interne (le site est un agencement de pages) et externe (le site établit des liens avec l'extérieur), dont les relations sont gérées par différents types d'instructions.

13 En principe, les concepteurs et les gestionnaires d'un site s'efforcent de maintenir une certaine cohérence entre la scénographie verbale et les divers composants de cette scénographie numérique. Ce qui doit permettre de stabiliser un ethos relativement unifié. Un blog intimiste par exemple va privilégier certaines couleurs, un certain type de découpage de la page, opter pour une iconographie suggestive, éviter les liens internes et externes à visée didactique, etc.

14 L'affaiblissement de la scène générique sur le Web se manifeste également sur le plan de la textualité. La conception classique du genre de discours associe chaque texte - considéré comme une totalité stabilisée qu'on peut appréhender globalement - à un genre. L'internaute, lui, est confronté non à un texte, mais à un écran qui se présente comme une mosaïque plus ou moins dense de modules hétérogènes, ce qui interdit de mettre en correspondance simple un texte et une scène d'énonciation. Dans cette mosaïque, on peut trouver des textes au sens habituel du terme mais aussi des listes, des diagrammes, des slogans, des titres, des vidéos, des bannières, etc. En outre, ce qui apparait sur l'écran n'est qu'un état transitoire; la plupart des modules sont renouvelés mais à des rythmes différents, en fonction du type de site concerné. Enfin, l'écran ne propose qu'une vue partielle d'une totalité qui ne se donne jamais intégralement : il y a divergence entre les scansions d'Internet et la pagination de l'imprimé. On sait aussi que l'hypertextualité implique une « lecture » qu'on peut dire navigante, le texte étant en fait le produit contingent du parcours de l'internaute, qui fabrique l'hypertexte qu'il « lit». 

l'ethos n'est pas au centre de l'attention du producteur. Ainsi, dans le cas des annonces des sites de rencontre, l'ethos discursif est la visée même de l'énoncé, qui s'attache à montrer la personnalité de l'énonciateur. Considérons par exemple cette annonce, extraite du site Meetic (Maingueneau 2015 : 293), d'une femme qui adopte le pseudo de Lola472 (38 ans) :

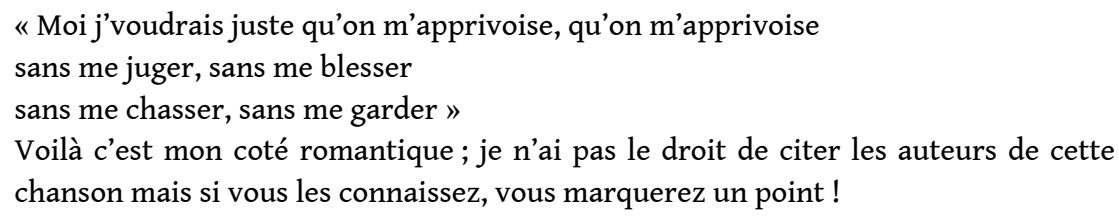

\subsection{La focalisation sur l'ethos}

On peut distinguer les énoncés qui sont focalisés sur la production d'un ethos et ceux où 
Sinon, je suis une fille tres indépendante, qui aime le sport (pour s'amuser) les voyages (pour les autres cultures) et la musique (qd mon cœur bat au rythme de la batterie et mes tempes à celui de la basse) et j'ai besoin sur ces plans là de quelqu'un qui me ressemble. Pour si vous etes encore curieux, anti conformistes, optimistes, réalistes, rêveurs.......

oui je sais ça fait beaucoup......

21 dans le paragraphe central (de «Sinon, je suis... » à «me ressemble »). Mais il s'efforce aussi de montrer un ethos personnel, comme on le voit à divers indices : le recours, au début du texte, à une citation littéraire, le maniement des parenthèses à fonction métaénonciative, le jeu avec les signifiants (" conformiste, optimiste, réaliste »), le tout porté par une énonciation qui maintient un équilibre délicat entre écrit et oral spontané. L'ethos est censé montrer en acte la conjonction harmonieuse, chez l'énonciatrice, de qualités complémentaires : une personne à la fois réaliste et imaginative, prosaïque et littéraire, sérieuse et fantaisiste, etc.

Cette focalisation sur l'ethos est caractéristique, à des degrés variables, de l'ensemble des pages personnelles. Par exemple, la typologie des scénographies qu'établit Lotta Lehti (2011) pour les blogs des hommes ou femmes politiques français apparait étroitement liée à la construction de leur ethos, essentielle pour des professionnels de la politique. Et ceci d'autant plus que ces blogs sont bien souvent gérés par des experts en communication. Mais si l'on considère par exemple les corpus de sites commerciaux ou administratifs, la mise en évidence d'un ethos personnel peut difficilement être la préoccupation majeure de leurs concepteurs. Ce qui ne signifie évidemment pas qu'il n'y ait pas de toute façon un certain ethos qui ressorte des signes de natures diverses disséminés sur l'écran, en particulier en termes de clarté, de simplicité, de commodité, etc.

\subsection{Ethos encadrant et ethos encadré}

Pour caractériser un site que l'on appréhende globalement, on peut parler d'ethos encadrant, qui imprègne de manière diffuse chacun des modules qu'il contient. Le rapport entre cet ethos encadrant et l'éthos des modules qu'il intègre est très variable. La situation la plus simple est quand les modules et leur encadrement sont gérés par la même instance productrice ; c'est le cas par exemple dans de nombreux blogs personnels. À l'opposé, on peut évoquer les sites qui renferment des modules très nombreux, hétérogènes, où de multiples intervenants insèrent leur contribution dans un cadre préétabli ; c'est le cas par exemple dans les sites d'information. Entre ces deux extrêmes il existe toutes sortes d'intermédiaires.

La notion d'encadrement est toutefois trop floue. Il faut en effet distinguer au moins trois niveaux en interaction constante : a) celui associé au logiciel avec lequel est conçu le site, b) celui qui résulte de la conception du site, c) celui des choix locaux (noms des rubriques, taille et police des titres, taille des textes, etc.). Chacun de ces niveaux est sources d'ethos; le moins riche étant celui du logiciel. J’ai ainsi pu évoquer (Maingueneau 2013) l'exemple de deux blogs très différents (celui d'une prostituée et celui d'un club de football rural) qui utilisent le même logiciel sans que cela induise le même ethos. 


\subsection{Textes et interventions}

Parmi les modules à dominante verbale que renferme un site, on peut opposer les textes au sens traditionnel du terme (formés de plusieurs phrases et relevant d'un genre identifié) et les interventions (Roulet 1981) associées qui s'inscrivent dans une chaîne communicationnelle que le locuteur n'a pas initiée. Considérons ce début d'article du site de Libération, à la rubrique « Désintox ».

\section{DESINTOX}

Invité sur France Info, Pierre Gattaz a cumulé les approximations sur la durée du temps de travail et l'impact de la suppression éventuelle de deux jours fériés «Deux jours fériés supprimés permettraient de faire deux jours [de plus] sur 225 jours, c'est-à-dire $1 \%$ de travail en plus par salarié [avec à la clé] entre 0,5 et $1 \%$ de PIB » en plus.

INTOX. Mercredi, Pierre Gattaz était invité sur France info. Bilan : il a ressorti une tarte à la crème cuisinée à sa sauce (le temps de travail des Français), et une promesse bidon (une croissance dopée par la suppression de deux jours fériés). « Il faut dire aux Français que nous travaillons 1660 heures par an alors que la moyenne européenne et les Allemands, c'est 1850. Nous travaillons 200 heures de moins par personne, ce qui est énorme », a commencé le patron des patrons. Avant d'ajouter : «Deux jours fériés supprimés permettraient de faire deux jours [de plus] sur 225 jours, c'est-à-dire $1 \%$ de travail en plus par salarié ", avec à la clé « entre 0,5 et $1 \%$ de PIB » en plus.

DESINTOX. Commençons par le temps de travail. Comme souvent sur ce sujet complexe, où les sources sont aussi nombreuses que les manières de les interpréter, Gattaz prend le chiffre qui lui sied... et lui fait dire ce qu'il ne dit pas. Le chiffre de 1660 heures de travail qu'il cite existe bel et bien. Il provient de la dernière étude sur le sujet de Coe-Rexecode, réalisée avec Eurostat. On y lit bien que la durée effective annuelle du temps de travail est de 1661 heures en France. À bonne distance des Allemands (1847) et de la moyenne européenne. Seule la Finlande travaille moins.

Peut-on pour autant, sur cette base, affirmer qu'en France, "nous travaillons 200 heures de moins par personne? » Non.

Comme indiqué dans l'étude, le chiffre concerne uniquement les salariés à temps plein, à l'exclusion des travailleurs indépendants et surtout des salariés à temps partiel. Un choix, que Gattaz se garde bien de préciser, et qui change pas mal de choses, d'autant plus que le temps partiel (qu'une étude de l'Insee assimilait, dans certains cas, à une forme de partage du temps de travail) est d'ampleur très variable dans les différents pays. En témoigne l'exemple de l'Allemagne, invoqué par Pierre Gattaz. Les Allemands sont plus fréquemment à temps partiel que les Français $(27,3 \%$ des salariés contre $18,4 \%)$. Et les salariés à temps partiels français travaillent en moyenne davantage (993 heures) que les travailleurs à temps partiel allemands (851 heures) [.... $]^{4}$

Cédric Mathiot et Juliette Deborde INTOX (avec en particulier le recours à un lexique familier et les variations de plans
énonciatifs), et un ethos d'expert qui domine dans la partie « Désintox ». On a affaire à un
ethos hybride aisément identifiable, caractéristique du positionnement du journal.

Si maintenant nous nous tournons vers les commentaires qui suivent cet article, il est très difficile de faire émerger de ces interventions un ethos consistant. On a affaire à des bribes d'écrits en style parlé, comme il est de règle dans ce type d'énoncés.

COMMENTAIRES 
- pipopatakes 26 septembre 2014 à 10:21

Quand Yvon Gattaz, pardon Pierre, le fils parle, j'ai toujours l'impression qu'il vient de sortir d'une soirée bien arrosée (son père c'était pareil).

On comprend rien, il dit n'importe quoi, tout et son contraire... bref, en un mot, le patron des patrons!

et moins les employés gagnent, plus c'est dans la poche du patron.

Un gars disait, travaillez plus pour gagner plus encore! Ouais, depuis, les employés

gagnent bien moins, et les patrons bien plus.

- alain31 26 septembre 2014 à 9:53

Ce mec sera satisfait quand nous bosserons comme au Bangladesh.

- seppel84 26 septembre 2014 à 9:33

Faire la moyenne en mélangeant les temps partiels et les temps complets relève de

la mauvaise foi. Dans le droit fil de la rhétorique antique, les études en termes d'ethos privilégient en général les locuteurs en chair et en os, pleinement individués. La réalité, on le sait, est beaucoup plus complexe. Et particulièrement sur le Web. C'est ainsi que certains sites associés au nom d'un individu sont en fait le masque d'une institution. Le «blog de Mélanie ", par exemple ${ }^{5}$, bien qu'il exhibe la photo d'une jeune femme censée être Mélanie, est la vitrine de la faculté de Droit de Montpellier I. Le site n'aurait pas été différent s'il s'était intitulé : « Site de la faculté de Droit de Montpellier I ».

Fig.1. Un blog institutionnel

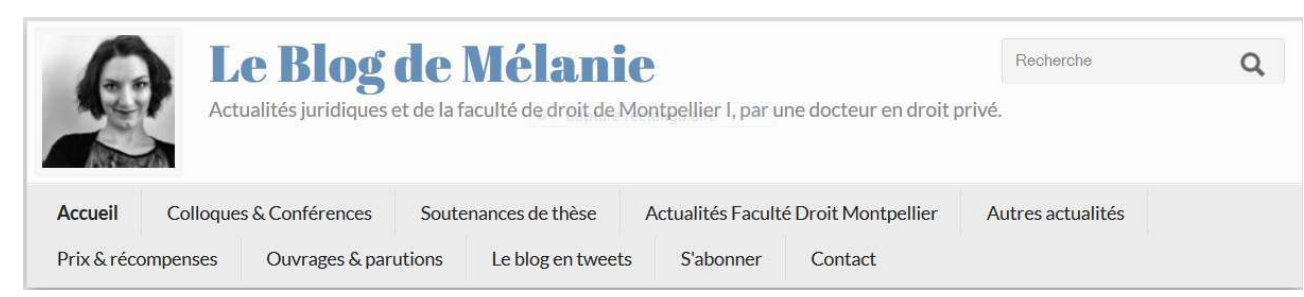

Les sources d'énonciation peuvent avoir un degré d'incarnation très variable. Mais il est fort difficile d'en dresser une typologie à partir des étiquettes usuelles. Si par exemple on parle d'« ethos de marque », on recourt à une catégorie qui en fait peut recouvrir des réalités très diverses. Dans le cas de la publicité, la marque est associée à un ethos fortement anthropomorphe, dont la dimension "expérientielle» est saillante. En revanche, dans le cas d'un site de commerce, la marque n'est pas en règle générale associée à un ethos identifiable. Le site se veut en effet avant tout fonctionnel : des termes évaluatifs tels que "convivial ", " compliqué », « confus », etc. caractérisent le mode de circulation dans le site, et non la source.

On peut néanmoins distinguer différents types de sources énonciatives, en fonction du type d'ethos qu'ils favorisent, en gardant à l'esprit que ce ne sont pas des catégories tranchées : les journaux en ligne par exemple participent à la fois de l'ethos collectif et de l'ethos de marque, les sites d'université associent ethos institutionnel et ethos de marque, 
etc. Parmi ces ethè on peut distinguer deux ensembles : le premier se caractérise par la saillance, le second par l'effacement de l'ethos. J'ai introduit la notion d'effacement d'ethos (Maingueneau 2014) sur le modèle de l'« effacement énonciatif» (Vion 2001, Rabatel 2004), qui désigne la stratégie consistant pour le locuteur à « donner l'impression "qu'il se retire de l'énonciation", qu'il "objectivise" son discours en "gommant" non seulement les marques les plus manifestes de sa présence (les embrayeurs) mais également le marquage de toute source énonciative identifiable » (Vion 2001 : 334).

\section{a) Saillance d'ethos}

32 - L'ethos personnel est celui d'un individu porteur d'un nom propre; doté d'une « corporalité » et d'un « caractère ", il peut jouer pleinement le rôle d'un "garant » pour l'énoncé. Il prospère sur les blogs personnels et les sites sociaux. On est toutefois obligé de distinguer entre l'ethos personnel extradiscursif (l'individu est identifié dans le monde extérieur au Web) et l'ethos personnel purement discursif, c'est-à-dire l'ethos d'individus qui n'existent que comme le corrélat de leurs énoncés (cas des pseudos). Ce phénomène débouche naturellement sur l'hétéronymie, la possibilité pour le même individu extradiscursif de se doter sur le Web de diverses personnalités.

33 - L'ethos collectif (un parti, une association, un syndicat, etc.) conserve un certain nombre de traits de l'individu, dans les trois dimensions de l'ethos: catégorielle, expérientielle et idéologique.

34 - L'ethos de marque émerge de la communication faite par la marque. Ce qui pour une bonne part différencie les formes innovantes de la publicité contemporaine et les formes plus traditionnelles, c'est précisément de s'attacher prioritairement à conférer un ethos à la marque, au lieu de se focaliser sur les qualités des produits vendus.

\section{b) Effacement d'ethos}

35 Le recours à l'effacement d'ethos ne signifie pas que le site ne produit pas d'ethos (toute production sémiotique rapportée à une source implique un ethos), mais seulement que la source s'efface derrière la gestion des parcours des usagers (voir par exemple le site www.impots.gouv.fr' qui, comme son nom l'indique, est dédié au paiement des impôts). Nous parlons ici de ce qui se passe au niveau de l'ensemble du site; cela n'exclut pas qu'un ou plusieurs modules soient associables à un ethos identifiable: logo, photos, message du directeur, etc. C'est ainsi que la page d'accueil du site de l'université Paris-Est Créteil (http://www.u-pec.fr/ ${ }^{7}$ ) en octobre 2014 donnait accès à un entretien avec le président, intitulé « Vers une nouvelle université ».

Cet effacement d'ethos concerne aussi bien les sites institutionnels (ministères, mairies, universités, etc.), les sites commerciaux ou pornographiques (Perea 2012) qui tendent, à des titres différents, à la pure fonctionnalité. Certes, ils sont associés à un nom propre de marque ou d'institution, mais ils cherchent à montrer qu'ils répondent le plus efficacement possible aux besoins des internautes. Ils placent ainsi au premier plan les découpages et les instruments qui doivent faciliter la navigation du visiteur, en fonction de ses motivations probables.

Néanmoins, quand s'exerce une véritable concurrence, ces sites sont soumis à deux injonctions simultanées qui ne sont pas toujours faciles à concilier. D'une part, ils tendent à un effacement d'ethos en privilégiant la fonctionnalité; d'autre part, ils sont soumis à 
une logique d'image de marque, qui les incite à produire un ethos identifiable. Les pages d'accueil du site de l'université Paris-Est Créteil $^{8}$ comme de celui de l'université Paris IV ${ }^{9}$ par exemple sont organisées de manières comparables : nom et logo, choix de la langue, cadre pour la fonction "recherche", rubriques en listes horizontales et verticales, opposition entre une partie stable et une partie variable ("à la Une »), etc. Mais sur ces pages on peut aussi être sensible aux indices de tous ordres qui témoignent d'une volonté de produire un ethos caractéristique.

Fig. 2. La page d'accueil de l'université Paris-Est Créteil Val de Marne

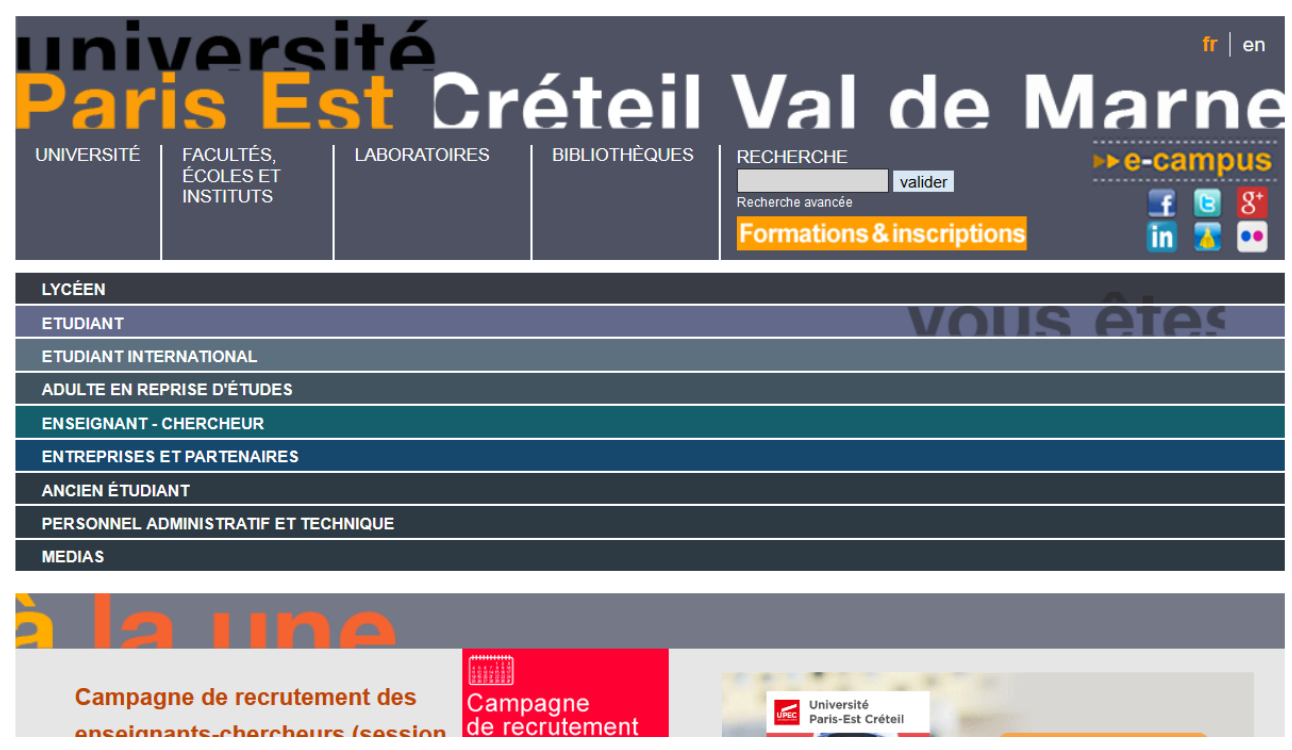

Fig. 3. La page d'accueil de l'université Paris IV

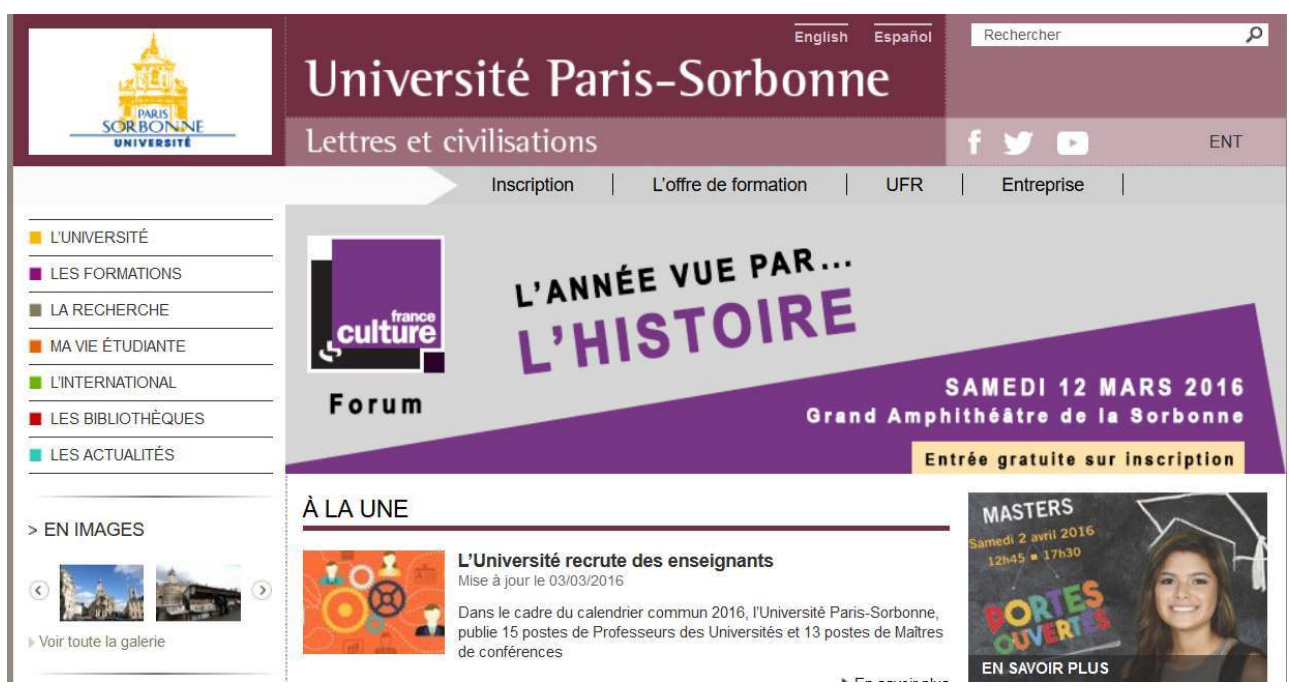

Cette double injonction est bien soulignée dans la recherche de C. Barats, J.-M. Leblanc et P. Fiala (2013) qui ont étudié par des méthodes quantitatives l'évolution des pages d'accueil de 21 établissements d'enseignement supérieur franciliens entre 2007 et 2011 en prenant en compte à la fois le texte, les images et la composition des pages. Ils ont abouti à la conclusion que ces sites se ressemblent de plus en plus mais qu'ils cherchent aussi à produire un ethos identifiable: "cette visualisation des résultats tend à confirmer l'émergence de standards dans la rhétorique de l'accueil académique tout en soulignant 
l'importance que les universités accordent à la présentation de soi et aux facteurs de différenciation » (2013:114). On voit ainsi apparaitre « des logiques de communication et de marque, jusqu'alors absentes du champ académique » (Ibid. : 117).

\section{Conclusion}

L'ethos reste pour le moment une catégorie d'analyse insuffisamment spécifiée pour être à la mesure de la diversité des situations de communication où il est impliqué. Tant qu'il s'agissait d'un concept purement rhétorique, dont la pertinence était cantonnée à l'art oratoire, un type de " preuve » au service d'une stratégie argumentative univoque, on est demeuré peu conscient de son contenu exact et de ses limites. En étendant à partir des années 1980 son champ de validité à l'ensemble des productions discursives, l'analyse du discours s'est dotée d'un outil précieux, mais a progressivement été amenée à s'interroger sur les problèmes que soulève son application à des corpus différents de ceux pour lesquels il a été conçu. Tant qu'il s'agit de textes relevant d'une généricité "classique », il est possible de minimiser ces problèmes; mais cela devient impossible quand on se confronte au Web, qui déstabilise un certain nombre de présupposés immémoriaux quant à la textualité et à l'auctorialité. C'est donc un vaste chantier qui s'ouvre.

\section{BIBLIOGRAPHIE}

Amossy, Ruth, 2011, La Présentation de soi. Ethos et identité verbale, Paris, PUF.

Aristote, 1967, Rhétorique, trad. M. Dufour, Paris, Les Belles Lettres.

Auchlin, Antoine, 2001, « Ethos et expérience du discours : quelques remarques », dans M. Wauthion et A.-C. Simon (dir.), Politesse et idéologie. Rencontres de pragmatique et de rhétorique conversationnelle, Louvain, Peeters, p. 75-93.

Barats, Christine, Leblanc, Jean-Marc et Fiala, Pierre, 2013, « Approches textométriques du Web : corpus et outils », dans C. Barats (dir.), Manuel d'analyse du Web, Paris, Armand Colin, p. 100-124.

Charaudeau, Patrick, 2005, Le Discours politique. Les masques du pouvoir, Paris, Vuibert.

Ducrot, Oswald, 1984, Le Dire et le Dit, Paris, Minuit.

Fairclough, Norman, 2003, Analyzing Discourse. Textual Analysis for Social Research, Londres, Routledge.

Lehti, Lotta, 2011, « Blogging Politics in Various Ways: A typology of French Politicians' Blogs », Journal of Pragmatics, vol. 43, n 6, p. 1610-1627.

Maingueneau, Dominique, 1984, Genèses du discours, Liège, Mardaga.

Maingueneau, Dominique, 1993, Le Contexte de l'œuvre littéraire, Paris, Dunod.

Maingueneau, Dominique, 1998, Analyser les textes de communication, Paris, Dunod. 
Maingueneau, Dominique, 2000, « Lecture, incorporation et monde éthique », Études de linguistique appliquée, $\mathrm{n}^{\circ} 119$, p. 265-276.

Maingueneau Dominique, 2013, «Genre de discours et Web : existe-t-il des genres Web ? ", dans

C. Barats (dir.), Manuel d'analyse du Web en sciences humaines et sociales, Paris, Armand Colin, p. 74-93.

Maingueneau, Dominique, 2014, « Retour critique sur l'ethos », Langage et société, n 149 , p. 31-48.

Maingueneau, Dominique, 2015, «De l'ethos au style : la présentation de soi sur les sites de rencontre », dans E. Bordas et G. Molinié (dir.), Style, langue et société, Paris, Champion, p. 283-298.

Perea, François, 2010, « L'identité numérique : de la cité à l'écran. Quelques aspects de la représentation de soi dans l'espace numérique ", Les Enjeux de l'information et de la communication, 1/ 2010, p. 144-159.

Perea, François, 2012, «Les sites pornographiques par le menu : pornotypes linguistiques et procédés médiatiques ", Genre, sexualité et société, $\mathrm{n}^{\circ} 7$, mis en ligne le $1^{\mathrm{er}}$ juin 2012, [En ligne], http://gss.revues.org/2395, consulté le 26 septembre 2014.

Rabatel, Alain, 2004, « L'effacement énonciatif dans les discours rapportés et ses effets pragmatiques ", Langages, $n^{\circ} 156$, p. 3-17.

Roulet, Eddy, 1981, «Échanges, interventions et actes de langage dans la structure de la conversation », Études de linguistique appliquée, $n^{\circ}$ 44, p. 7-39.

Vion, Robert, 2001, «Effacement énonciatif et stratégies discursives ", in De la syntaxe à la narratologie énonciative, dans M. De Mattia, et A. Joly (dir.), Gap-Paris, Ophrys, p. 331-354.

Woerther Frédérique, 2007, L'Ethos aristotélicien. Genèse d'une notion rhétorique, Paris, Vrin.

\section{NOTES}

1. C'est moi qui souligne.

2. Un «hypergenre » n'est pas un genre mais un formatage aux contraintes pauvres (lettre, dialogue, entretien, etc.) qui peut recouvrir des genres très divers. Cette notion a reçu d'autres noms; ainsi N. Fairclough parle-t-il de «disembedded genre» (2003: 68), qu'il oppose aux « situated genres ".

3. On a appris le 2 octobre 2014 que Facebook autorisait désormais ses abonnés à utiliser des noms d'emprunt. Officiellement, c'est pour ne pas blesser « la communauté des drag queens et kings, des transgenres, et le groupe plus large de nos amis, de nos voisins, et des membres de la communauté LGBT, selon les termes de l'entreprise, car ces personnes utilisent constamment des pseudos dans la "vraie vie" " (http://www.lefigaro.fr/flashactu/2014/10/02/97001-20141002FILWWW00020-facebook-va-assouplir-ses-regles-sur-l-

identite.php, consulté le 2 octobre 2014). Mais il est clair que la logique même du web va dans le sens d'une dissociation entre identité sociale et identité numérique.

4. http://www.liberation.fr/politiques/2014/09/26/au-medef-l-intox-ne-connait-pas-de-jourferie_1108091 (consulté le 26 septembre 2014).

5. http://leblogdemelanie.over-blog.com/ (consulté le 26 septembre 2014).

6. Consulté le 15 octobre 2014.

7. Consulté le 3 octobre 2014.

8. http://www.u-pec.fr/ (consulté le 8 mars 2016).

9. http://www.paris-sorbonne.fr/ (consulté le 8 mars 2016). 


\section{RÉSUMÉS}

Dans cet article je réfléchis sur les problèmes que pose le Web quand on l'étudie à l'aide de la catégorie de l'ethos discursif. La conception classique de la généricité textuelle - dont l'ethos est traditionnellement solidaire - n'est pas adaptée au fonctionnement du Web. Pour mieux prendre en compte sa spécificité, je fais intervenir divers paramètres : la focalisation ou non des énoncés sur l'ethos, la distinction entre ethos « encadrant » et " encadré », la différence entre les textes proprement dits et leurs commentaires, la possibilité d'effacer l'ethos.

In this article, I reflect on the problems that raise by when one studies the web with the help of the category of discursive ethos. The classical conception of text genres-from which traditionally ethos is inseparable-is not suited for the way the web functions. To take better into account its specificity, various parameters are mentioned: the focus of texts on ethos, the distinction between "framing" and "framed" ethos, the difference between texts and comments, the possibility of erasing ethos.

\section{INDEX}

Mots-clés : analyse du discours, ethos discursif, Web, genre de discours, hypergenre, scénographie iconotextuelle, scénographie réticulaire

Keywords : discourse analysis, discursive ethos, genre of discourse, iconotextual scenography

\section{AUTEUR}

\section{DOMINIQUE MAINGUENEAU}

Université Paris-Sorbonne 